\title{
Über die Wirkung von Gibberellin auf Wachstum und Blütenbildung bei verschiedenen Temperatur- und Licht-Verhältnissen
}

\author{
Von RÜDIGER KNAPP \\ (Z. Naturforschg. 11 b, 698-704 [1956]; eingegangen am 23. Mai 1956) \\ - Aus der Division of Biology, Earhart Plant Research Laboratory, \\ California Institute of Technology, Pasadena, California
}

\begin{abstract}
Die Gibberelline sind erst seit relativ kurzer Zeit bekannt. Sie können in geeigneten Konzentrationen insbesondere das Längenwachstum der Sprosse in sehr starkem Maße beschleunigen. Ihre Wirkung wurde unter dem Einfluß verschiedener Temperatur- und Lichtverhältnisse an Agrostemma githago und Galinsoga parviflora untersucht. Bei beiden Arten ist die Dauer der täglichen Belichtung von wesentlich stärkerem Einfluß auf die Wirkung von Gibberellin als Unterschiede in der Temperatur. Galinsoga parviflora wird von Gibberellin stärker beeinflußt als Agrostemma githago. Bei Galinsoga wird außer dem Längenwachstum der Sprosse und dem Beginn der Blüte, die auch bei Agrostemma gefördert werden, die Form und anfangs die Größe der Blätter, im Dauerlicht ferner die Zahl der fertilen Früchte in bestimmter Hinsicht verändert.
\end{abstract}

$\mathrm{D}$ e Gibberelline sind zuerst von japanischer Seite bearbeitet worden (Zusammenstellungen der japanischen Literatur in den Arbeiten von $\mathrm{Stoll}{ }^{1}$, $\mathrm{S}$ todola und Mitarbb. ${ }^{2}$ ). Sie werden von dem Pilz Gibberella fuiikuroi (S a w.) Woll. gebildet. Eine Reihe von Bearbeitern sehen Fusarium moniliforme $\mathrm{S}$ held. als dessen imperfektes Stadium an (Brierle ${ }^{3}$ ). Gibberella verursacht eine Pflanzenkrankheit, die in Reispflanzungen auftritt (Bakanaë-Krankheit). Über den Stoffwechsel, einige Wachstumsbedingungen und biologisch wirksame Stoffe dieses Pilzes führte außer den japanischen Bearbeitern $\mathrm{S}$ to $1^{1}{ }^{1}$ Untersuchungen durch. Neuerdings haben sich auch Cross ${ }^{4}$, Curtis und Cross ${ }^{5}$, Mulholland und Ward ${ }^{6}$, Borrow, Brian und Mitarbb. ${ }^{7}$, S to dola und Mitarbb. ${ }^{2}$ mit der chemischen Struktur und Isolation dieser Verbindungen beschäftigt. Brian und Mitarbb. (Brian, Elson, Hem ming und Radley ${ }^{8}$, B rian und $\mathrm{Hem}$ min ${ }^{9}$ ) führten Untersuchungen über die wuchsfördernden Eigenschaften der Gibberellinsäure durch.

Die Untersuchungen, über deren Ergebnisse hier berichtet wird, mögen zur Kenntnis der Wirkung der Gibberelline auf das Wachstum und die Blüten-

1 C. S t o 11, Phytopathol. Z. 22, 233 [1954].

- F. H. S todola, K. B. R a p er, D. I. F e nnell, H. F. Conway, V. E. Sohns, C. T. L ang ford u. R. W. J a c k s o n, Arch. Biochem. Biophysics 54, 240 [1955].

3 W. B. Brierley, Appendix in E. Gäuman n, Principles of plant infection. S. 498, London 1950.

4 B. E. Cros s, J. chem. Soc. 1954, 4670.

5 P. J. Curtis u. B. E. Cross, Chem. and Ind. 1954, 1066 .

${ }_{6}$ T. P. C. Mulholland u. G. Ward, J. chem. Soc. 1954,4676 . bildung unter dem Einfluß verschiedener Temperatur- und Lichtbedingungen beitragen. Die Versuche wurden im Earhart Plant Research Laboratory, das hierfür besonders gute Möglichkeiten bot (W e n $\mathbf{t}^{\mathbf{1 0}}$ ), mit Agrostemma githago L. und Galinsoga parviflora C a v. durchgeführt.

\section{Arbeitsmethoden}

Das verwendete Präparat der Gibberelline A und X stammt von der Firma Merck u. Co., Inc., Rahway N. J. Die Gibberelline A $\left(\mathrm{C}_{22} \mathrm{H}_{26} \mathrm{O}_{7}\right)$ und $\mathrm{X}\left(\mathrm{C}_{19} \mathrm{H}_{22} \mathrm{O}_{6}\right)$ unterscheiden sich zwar trotz Verwandtschaft in ihrem chemischen Aufbau. Jedoch ist ihre physiologische Wirkung nach S todola und Mitarbb. ${ }^{2}$ ungefähr gleichartig. Gibberellin X ist identisch mit Gibberellinsäure.

Die Gibberelline wurden in einer kleinen Menge Äthylalkohol gelöst. Dann erfolgte eine starke Verdünnung mit Wasser. Die so gewonnenen Lösungen hatten einen Gehalt von 2\% Äthylalkohol. Ein solcher Alkoholgehalt hat bei den applizierten Mengen und Methoden keinen Einfluß auf das Wachstum, wie durch ergänzende Untersuchungen festgestellt wurde. Jedoch wurden vorsichtshalber auch die Kontrollpflanzen mit der gleichen Menge Lösung von Äthylalkohol in Wasser (2\%) ohne Gibberellin wie die anderen Serien behandelt. Die Lösungen wurden auf die jüngsten, voll entwickelten Blätter aufgetragen. Die Behandlung erfolgte dreimal an drei aufeinanderfolgenden Tagen. Jeden Tag erhielt je eine

7 A. B orrow, P. W. Brian, V. E. Chester, P. J. Curtis, H. G. Hemming, C. Henehan, E. G. J efferys, P. B. Lloyd, I. S. Nixon, G. L. F. Norris u. M. R a d ley, J. Sci. Food Agric. 6, 340 [1955].

8 P. W. B rian, G. W. Els on, H. G. H e m m ing u. M. R a d le y, J. Sci. Food Agric. 5, 602 [1954].

9 P. W. B rian u. H. G. Hemmi ng, Physiol. Plantarum 8, 669 [1955].

10 F. W. W e n t, Chronica Botanica 12, 89 [1950]. 
Pflanze $0,05 \mathrm{ccm}$. Die Lösungen waren so hergestellt worden, daß mit $0,15 \mathrm{ccm}$ die betreffenden Mengen von Gibberellin ( 3 und $9 \mu \mathrm{g}$ pro Pflanze) auf die Pflanzen gelangten. Das Gibberellin wurde offensichtlich gut von den Blättern aufgenommen und rasch in die wachsenden Teile der Pflanzen transportiert, wie die schnell einsetzende Wirkung zeigte. Die Mengen von Gibberellin pro Pflanze wurden auf Grund der Erfahrungen von B ri a n und $\mathrm{Hemming}{ }^{9}$ bei Erbsen so gewählt, daß ein nachhaltiger Effekt erwartet werden konnte.

Die Anzucht der Pflanzen und die Durchführung der Versuche erfolgte in Klimakammern mit konstanten Temperaturen, $75 \%$ relativer Luftfeuchtigkeit und in einer Lichtintensität von $7700 \pm 200$ Lux. Es wurde der Einfluß von drei verschiedenen Temperaturen, $26^{\circ}, 17^{\circ}$ und $10^{\circ} \mathrm{C}$, untersucht. Die Lichtverhältnisse wurden dadurch variiert, daß bei jeder dieser Temperaturen eine Serie täglich 16-stdg. Dunkelheit ausgesetzt war (Kurztag-Bedingungen). Die Pflanzen der anderen Serien wuchsen im Dauerlicht. Die Pflanzen wurden während des gesamten Versuches und der Anzucht in Vermiculite kultiviert, der ständig gleichmäßig mit einer Nährlösung feucht gehalten wurde, die in $1 l$ Wasser neben Spurenelementen nach $\mathrm{H}$ o a g l a $\mathrm{n} d$ folgende Verbindungen enthielt: 0,91 $\mathrm{Ca}\left(\mathrm{NO}_{3}\right)_{2} ; 0,544 \mathrm{~g} \quad \mathrm{MgSO}_{4} \cdot 7 \mathrm{H}_{2} \mathrm{O} ; 0,1552 \mathrm{~g} \quad \mathrm{KH}_{2} \mathrm{PO}_{4}$; $0,5532 \mathrm{~g} \mathrm{KNO}_{3} ; 0,05845 \mathrm{~g} \mathrm{NaCl}$. Die Arbeitsmethoden entsprachen also denen, die sich bereits bei anderen Experimenten für das Wachstum von Agrostemma githago als günstig erwiesen hatten ( $\mathrm{K} n$ a p p $\left.{ }^{11}\right)$.

Die Pflanzen stammen von Saatgut, das im Earhart Plant Research Laboratory geerntet wurde. Die Elternpflanzen waren selektioniert und wuchsen in den Gewächshäusern mit kontrollierten Temperaturen und natürlichem Licht (Elternpflanzen von Agrostemma bei $23^{\circ}$ von 8-16 Uhr und $17^{\circ}$ von 16-8 Uhr; Elternpflanzen von Galinsoga parviflora bei $20^{\circ}$ von $8-16$ Uhr und $14^{\circ}$ von 16-8 Uhr).

Die Aussaat und Jugendentwicklung erfolgte im Dauerlicht, wobei Agrostemma Temperaturen von $7^{\circ}$, Galinsoga von $23^{\circ}$ ausgesetzt waren. $24 \mathrm{Stdn}$. vor der ersten Applizierung der Gibberellin-Lösung wurden die Pflanzen in die Klimakammern mit den Temperatur- und Lichtverhältnissen umgestellt, in denen die betreffenden Experimente durchgeführt wurden. Tab. 1 enthält Angaben über die Größe der Pflanzen zu diesem Zeitpunkt. Der mittlere Fehler zeigt hierbei die Variabilität des Materiales. Er wurde für je 50 Pflanzen berechnet.

\section{Wirkung auf das Sproßwachstum}

Am deutlichsten ist der Einfluß auf das Wachstum des Sprosses. Die mit Gibberellin in wirksamen Konzentrationen behandelten Pflanzen haben ein rascheres Höhenwachstum als die anderen Individuen. Erst nach einem bestimmten Zeitraum klingt die Wirkung ab. Untersuchungen in kurzfristigeren Abständen erlauben eine nähere Analyse der Wachstumsförderung unter den einzelnen Temperatur- und Lichtbedingungen. Für die beiden untersuchten Ar-

11 R. K n a p p, Naturwissenschaften 43, 115 [1956].

\begin{tabular}{|l|c|c|}
\hline & $\begin{array}{c}\text { Höhe } \\
\text { des Sprosses } \\
{[\mathrm{mm}]}\end{array}$ & $\begin{array}{c}\text { Länge des } \\
\text { längsten Blattes } \\
{[\mathrm{mm}]}\end{array}$ \\
\hline Agrostemma githago & $34,0 \pm 0,87$ & $91,2 \pm 1,23$ \\
Galinsoga parviflora & $59,5 \pm 0,98$ & $34,1 \pm 0,65$ \\
\hline
\end{tabular}

Tab. 1. Angaben über die Größe der Pflanzen.

ten zeigt je eine Abbildung die Höhe der Kontrollpflanzen (nicht mit Gibberellin behandelt) zu den verschiedenen Zeitpunkten und die Förderung des täglichen Zuwachses durch Gibberellingaben. Bei den Messungen wurde als Sproßhöhe der Zwischenraum zwischen dem Cotyledonarknoten und dem höchsten zu den einzelnen Zeitpunkten jeweils entwickelten Knoten berücksichtigt. Die Länge des Hypokotyles wurde nicht eingeschlossen, da diese zu sehr von den Pflanzbedingungen beeinflußt war.

Bei Agrostemma githago (Abb. 1 und 2) setzt die Wirkung im Dauerlicht rasch ein. In den ersten Tagen erfolgt hier die Hauptwirkung des Gibberellins. Danach tritt ein rascher Abfall der Förderung des Höhenwachstums ein. Nach etwa 8 Tagen wachsen daher die mit Gibberellin behandelten Pflanzen im allgemeinen nur wenig schneller oder gar langsamer als die Kontrollen. Im Kurztag ist die Wirkung des Gibberellins bei Agrostemma in den ersten Tagen nach der Behandlung relativ gering. Um den 5. Tag herum erfolgt unter diesen Bedingungen die maximale Förderung des Sproßwachstums. Im allgemeinen ist die Beschleunigung des täglichen Zuwachses in diesem Zeitraum höher als im Dauerlicht. Die Wirkung des Gibberellins hält bei 8-stdg. täglicher Belichtung länger an. Der Gegensatz zwischen dem Einfluß im Dauerlicht und im Kurztag ist bei Agrostemma githago viel größer als derjenige zwischen den einzelnen Temperaturen. Der letztere ist erstaunlich gering. Sowohl bei hohen als auch bei tiefen Temperaturen hat Gibberellin eine starke Wirkung. Im Dauerlicht liegt die Zeit der Förderung durch Gibberellin vor der Periode des größten Streckungswachstumes der Kontrollen. Im Kurztag ist das Wachstum der unbehandelten Pflanzen während des Untersuchungs-Zeitraumes ziemlich gleichmäßig (Abb. 2).

Bei Galinsoga parviflora (Abb. 3 und 4) setzt die Wirkung des Gibberellins im Dauerlicht ziemlich rasch ein, ohne allerdings so stark auf die ersten Tage nach der Applizierung konzentriert zu sein wie bei Agrostemma. Am 4. bis 7. Tage nach Beginn der Behandlung ist der Zuwachs bei $26^{\circ}$ und $17^{\circ}$, am 7 . 
bis 9 . Tage bei $10^{\circ}$ im Dauerlicht am meisten gefördert. Auch das Abklingen der Wirkung erfolgt in hohen Temperaturen sehr viel rascher als bei ge-
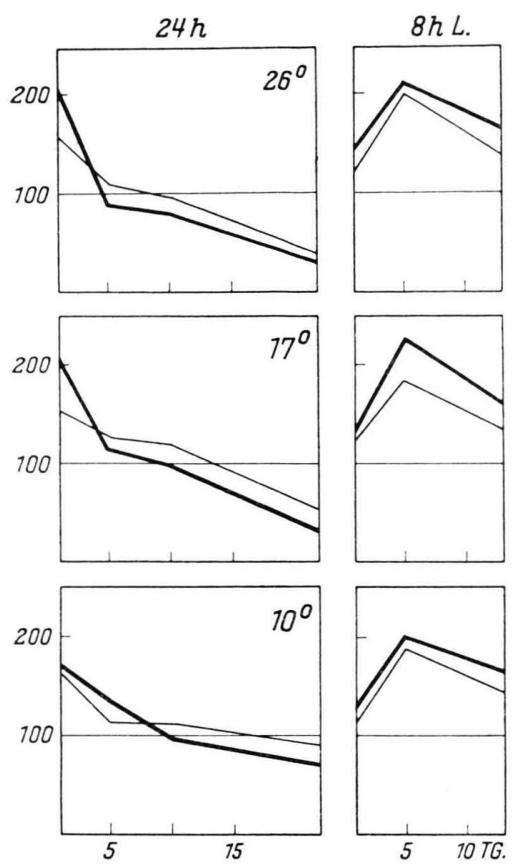

Abb. 1. Täglicher Zuwachs von Agrostemma githago bei verschiedenen Temperaturen. Links Zuwachs im Dauerlicht, rechts bei 8-stdg. täglicher Belichtung. Abszisse: Tage nach Beginn der Behandlung mit Gibberellin. Ordinate: Relativer täglicher Zuwachs (Zuwachs der Kontrollen $=100)$. Dicke Kurven: $9 \mu \mathrm{g}$ Gibberellin pro

Pflanze. Dünne Kurven: $3 \mu \mathrm{g}$ Gibberellin pro Pflanze.
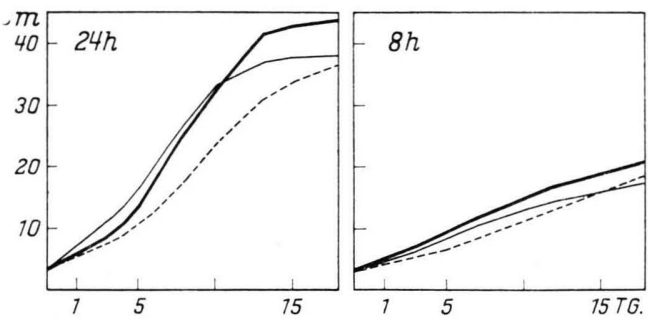

Abb. 2. Wachstumsveriauf der Sprosse von Agrostemma githago (nicht mit Gibberellin behandelte Kontrollen) in 3 verschiedenen Temperaturen im Dauerlicht (links) und bei 8 -stdg. täglicher Belichtung (rechts). Abszisse: Tage nach Beginn der Behandlung der anderen Serien mit Gibberellin. Ordinate: Höhe der Sprosse in $\mathrm{cm}$. Dünne Kurven Wachstum bei $26^{\circ}$, dicke Kurven bei $17^{\circ}$, unterbrochene Kurven bei $10^{\circ}$.

ringerer Wärme. Etwa vom 14. Tage ab ist bei $26^{\circ}$ der Zuwachs bereits geringer als bei den unbehandelten Pflanzen. Bei $10^{\circ}$ hält dagegen die Wuchsförderung bis zum Abschluß der Messungen an. Dieser unterschiedliche Einfluß bei den einzelnen Tempe-

12 J. Th. de H a a n, Phytopathol. Z. 10, 235 [1937]. raturen stellt in gewissem Sinne eine Parallele zu den Ergebnissen der Untersuchungen von De $\mathrm{H}$ a an ${ }^{12}$ an mit Fusarium moniliforme befallenen Reispflanzen dar, der ungleiche Wirkungen bei verschiedenen Bodentemperaturen fand.
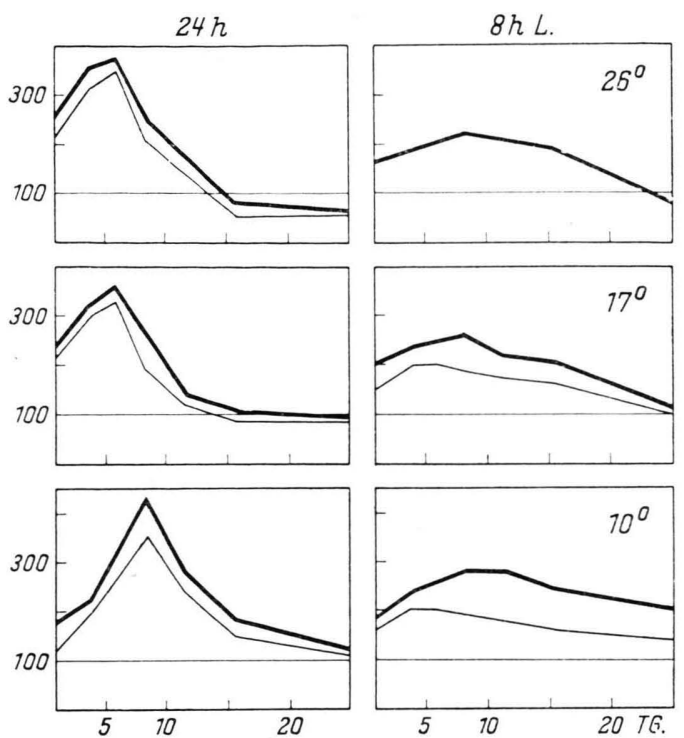

Abb. 3. Täglicher Zuwachs von Galinsoga parviflora bei verschiedenen Temperatur- und Lichtverhältnissen. Erläuterungen bei Abb. 1.
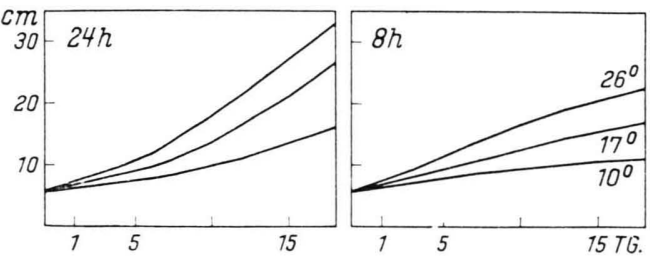

Abb. 4. Wachstumsverlauf der Sprosse bei Galinsoga parviflora (nicht mit Gibberellin behandelte Kontrollen) in verschiedenen Lichtverhältnissen und Temperaturen (obere Kurven bei $26^{\circ}$, mittlere Kurven bei $17^{\circ}$, untere

Kurven bei $10^{\circ}$ ). Weitere Erläuterungen bei Abb. 2 .

Im Kurztag setzt auch bei Galinsoga parviflora bei allen Temperaturen die Wirkung des Gibberellins im allgemeinen viel langsamer ein als im Dauerlicht und erreicht am 7. bis 12. Tage nach Beginn der Behandlung ihr Maximum. Die Wirkung hält auch länger an, erreicht jedoch nicht so hohe Werte in der Förderung des täglichen Zuwachses wie im Dauerlicht. Während im Dauerlicht diese Werte im Maximum bei allen Temperaturen über $350 \%$ liegen, überschreiten sie im Kurztag nicht 275 Prozent. Auch bei Galinsoga scheint für die Art der Wirkung des Gibberellins die tägliche Belichtungsdauer der Pflanzen von erheblich größerem Einfluß zu sein als die Höhe 


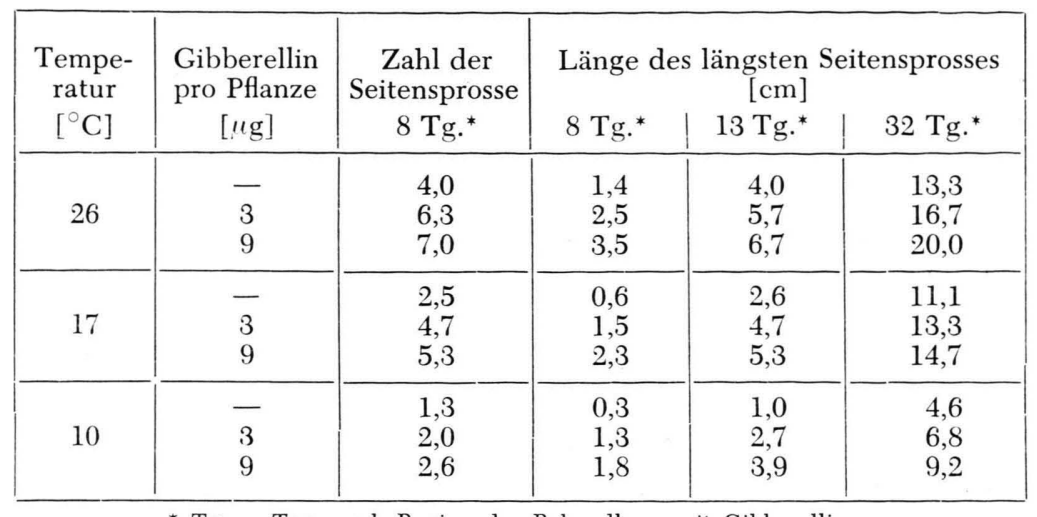

* Tg. = Tage nach Beginn der Behandlung mit Gibberellin.

Tab. 2. Seitensproßbildung bei Galinsoga parviflora unter dem Einfluß von Gibberellin im Dauerlicht.

\begin{tabular}{|c|c|c|c|c|c|c|c|c|}
\hline \multirow{2}{*}{$\begin{array}{c}\text { Tempe- } \\
\text { ratur } \\
{\left[{ }^{\circ} \mathrm{C}\right]}\end{array}$} & \multirow{2}{*}{$\begin{array}{l}\text { Licht } \\
\text { pro Tag } \\
\text { [Stdn.] }\end{array}$} & \multirow{2}{*}{$\begin{array}{c}\text { Gibberellin } \\
\text { pro Pflanze } \\
{[\mu \mathrm{g}]}\end{array}$} & \multicolumn{6}{|c|}{ Internodien } \\
\hline & & & 2. & 3. & 4. & 5. & 6. & 7. \\
\hline 26 & 24 & $\begin{array}{l}3 \\
9\end{array}$ & $\begin{array}{l}123 \\
107\end{array}$ & $\begin{array}{l}194 \\
172\end{array}$ & $\begin{array}{l}164 \\
164\end{array}$ & $\begin{array}{l}136 \\
147\end{array}$ & $\begin{array}{l}89 \\
99\end{array}$ & $\begin{array}{l}72 \\
84\end{array}$ \\
\hline 17 & 24 & $\begin{array}{l}3 \\
9\end{array}$ & $\begin{array}{l}136 \\
118\end{array}$ & $\begin{array}{l}157 \\
166\end{array}$ & $\begin{array}{l}133 \\
156\end{array}$ & $\begin{array}{l}86 \\
93\end{array}$ & $\begin{array}{l}66 \\
82\end{array}$ & $\begin{array}{l}54 \\
60\end{array}$ \\
\hline 10 & 24 & $\begin{array}{l}3 \\
9\end{array}$ & $\begin{array}{l}117 \\
107\end{array}$ & $\begin{array}{l}161 \\
203\end{array}$ & $\begin{array}{l}169 \\
207\end{array}$ & $\begin{array}{l}114 \\
129\end{array}$ & $\begin{array}{r}90 \\
110\end{array}$ & $\begin{array}{l}85 \\
91\end{array}$ \\
\hline 17 & 8 & $\begin{array}{l}3 \\
9\end{array}$ & $\begin{array}{l}158 \\
119\end{array}$ & $\begin{array}{l}171 \\
146\end{array}$ & $\begin{array}{l}131 \\
152\end{array}$ & $\begin{array}{l}104 \\
123\end{array}$ & $\begin{array}{l}80 \\
85\end{array}$ & $\begin{array}{l}42 \\
56\end{array}$ \\
\hline 10 & 8 & $\begin{array}{l}3 \\
9\end{array}$ & $\begin{array}{l}150 \\
110\end{array}$ & $\begin{array}{l}161 \\
136\end{array}$ & $\begin{array}{l}110 \\
160\end{array}$ & $\begin{array}{r}85 \\
103\end{array}$ & $\begin{array}{r}76 \\
103\end{array}$ & - \\
\hline
\end{tabular}

Tab. 3. Längen des 2.-7. Internodiums des Hauptsprosses von Galinsoga parviflora unter dem Einfluß von Gibberellin. Relative Werte (Längen bei unbehandelten Pflanzen $=100$ ). Das 2. ist das tiefste, das 7. das höchste, zuletzt gebildete der in der Tabelle berücksichtigten Internodien.

der Temperatur. Die Intensität der Wirkung des Gibberellins ist bei Galinsoga unter allen untersuchten Bedingungen höher. Denn bei Agrostemma wurden nur maximale Förderungen zwischen 200 und $230 \%$ gefunden.

Bei Galinsoga parviflora wurde auch die Seitensproßbildung erheblich durch Gibberellin beschleunigt (Tab.2). Sowohl deren Länge als auch deren Zahl nimmt anfangs mit wachsenden GibberellinGaben zu. Der Gegensatz in der Länge der Seitensprosse zwischen behandelten und unbehandelten Pflanzen ist jedoch bereits am 13. Tage nach der ersten Applizierung erheblich kleiner als zu einem früheren Zeitpunkt (8 Tage nach der ersten Behandlung).

Es erhebt sich die Frage, ob die Förderung des Sproßwachstumes mit Änderungen in den Größenverhältnissen der verschiedenen Internodien verbun- den ist oder ob sich die ausgewachsenen Sprosse der mit Gibberellin behandelten Pflanzen in dieser Hinsicht in keiner Weise von den Kontrollen unterscheiden. Im letzten Falle würde also nur eine allgemeine Wachstumsbeschleunigung vorherrschend sein, ohne daß später am Sproß Spuren der Wirkung des Gibberellins erkennbar wären. Das ist tatsächlich bei Agrostemma der Fall. Die Analysen der Länge der einzelnen Internodien ergaben bei dieser Art keine Unterschiede zwischen behandelten und unbehandelten Pflanzen.

Dagegen zeigen bei Galinsoga parviflora die Internodien, die sich unmittelbar nach der Einwirkung des Gibberellins entwickelten, größere Länge als diejenigen der unbehandelten Pflanzen (Tab.3). Unter jeder der berücksichtigten Temperatur- und Lichtverhältnisse ist zunächst die Förderung der Internodienlänge durch die schwächeren Giberrellingaben 
größer. Erst zwischen den höheren Knoten erreichen die Internodien der mit den stärksten Mengen von Gibberellin behandelten Pflanzen die größten Längen. Die später entwickelten Internodien sind dagegen bei den unter dem Einfluß der Gibberellin-Lösungen beider Konzentrationen stehenden Pflanzen kürzer als bei den Kontrollpflanzen. Bei den mit $3 \mu \mathrm{g}$ behandelten Pflanzen ist die Verminderung der Längen zunächst stärker und tritt oft auch bei früheren Internodien ein als bei den mit $9 \mu \mathrm{g}$ behandelten Individuen. Die maximalen Internodien-Verlängerungen betragen zwischen 150 und 210 Prozent. Sie sind also geringer als die Zuwachsförderungen (Abb. 3). Eine größere Förderung der Länge der Internodien ist aus den Ergebnissen der Experimente von $\mathrm{B}$ rian und $\mathrm{He}$ m min ${ }^{9}{ }^{9}$ mit Erbsen (Meteor) unter Gibberellinsäure-Einfluß zu erschließen.

Bei Pflanzen, die ihre Entwicklung abgeschlossen haben, konnte weder bei Agrostemma githago noch bei Galinsoga parviflora ein wesentlicher Unterschied in der Höhe des Sproß-Systemes zwischen den Kontrollen und den mit Gibberellin behandelten Serien festgestellt werden. Auch in der Anzahl und Länge der Seitensprosse bei Galinsoga waren keine deutlichen Verschiedenheiten zwischen den unbehandelten Pflanzen und den früher der Einwirkung des Gibberellins ausgesetzten Serien in diesem Entwicklungsstadium zu ermitteln. Das gleiche gilt für die Gesamtgewichte der Pflanzen.

\section{Wirkung auf die Blätter}

Die Blattform und Blattlänge wird bei Agrostemma githago kaum durch Gibberellin beeinflußt. Selbst in Zeiten stärkster Wirkung des Gibberellins auf das Höhenwachstum der Sproßachse konnten bei den Blättern keine wesentlichen Vergrößerungen der Länge festgestellt werden. Auch die Unterschiede im Chlorophyllgehalt, soweit sie aus der Intensität der Grünfärbung geschätzt werden können, waren nicht deutlich.

Die Unterschiede in der Zahl der entwickelten Blattpaare 8 Tage nach Beginn der Gibberellin-Behandlung bei Agrostemma githago zeigt Tab. 4 (relative Werte, Mittel aller Temperaturen):

\begin{tabular}{|c|c|c|}
\hline $\begin{array}{c}\text { Gibberellinmenge pro } \\
\text { Pflanze (Agrostemma) } \\
{[\mu \mathrm{g}]}\end{array}$ & Dauerlicht & $\begin{array}{c}\text { 8Stdn.Licht } \\
\text { täglich }\end{array}$ \\
\hline- & 100 & 100 \\
3 & 105 & 102 \\
9 & 110 & 105 \\
\hline
\end{tabular}

Tab.4. (Erläuterung im Text).
Es ergibt sich also eine schwache Förderung der Geschwindigkeit der Bildung der Blätter.

Wesentlich anders liegen die Verhältnisse bei $\mathbf{G a}$ linsoga parviflora. Hier bewirkt Gibberellin zunächst ein rascheres Längenwachstum der Blätter, wie aus Abb. $5 \mathrm{zu}$ ersehen ist. Dieser Einfluß ist aber nicht von Dauer. Am 32. Tage nach Einsetzen der Gibberellingaben waren bei den Bedingungen, die raschen Wuchs ermöglichen (Dauerlicht bei $26^{\circ}$ und $17^{\circ}$,
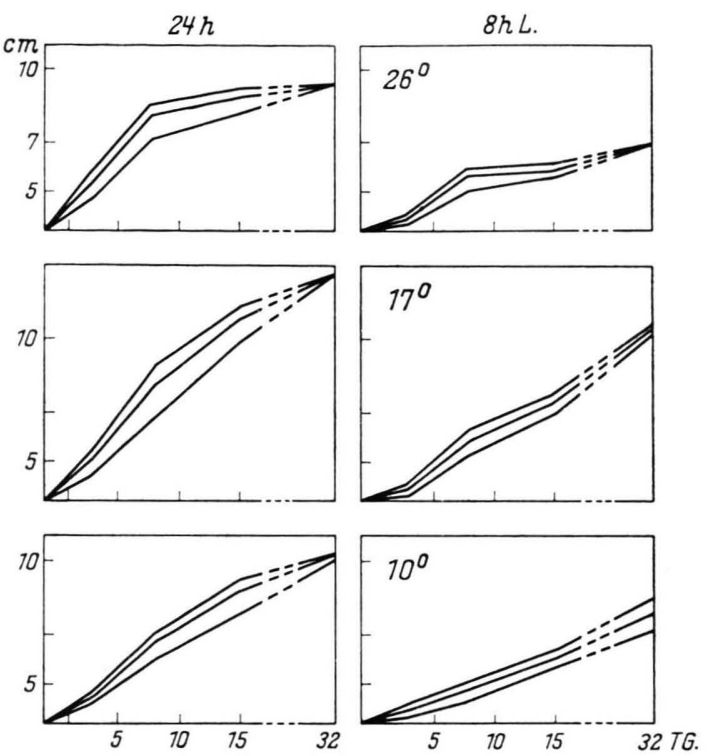

Abb. 5. Blattlängen von Galinsoga parviflora (Ordinate) an verschiedenen Tagen nach Beginn der Behandlung mit Gibberellin im Dauerlicht (links) und bei 8-stdg. täglicher Belichtung (rechts) bei 3 verschiedenen Temperaturen. Obere Kurven $9 \mu \mathrm{g}$ Gibberellin pro Pflanze; mittlere Kurven $3 \mu \mathrm{g}$ Gibberellin pro Pflanze; untere Kurven Kontrollen (unbehandelte Pflanzen).

Kurztag bei $26^{\circ}$ ), die längsten Blätter bei den Kontrollen und den behandelten Pflanzen bereits gleich. Inbesondere wird aber die Form der Blätter durch die Verbindung wesentlich beeinflußt. Unter der Wirkung des Gibberellins wurden die Blattspreiten viel schmäler. Bei 8-stdg. täglichem Licht bei $10^{\circ}$ ergaben sich folgende Quotienten für die Länge der Blattspreiten durch deren Breiten (erstes Blattpaar unter der am frühesten entwickelten Blüte):

$$
\begin{array}{ll}
\text { Unbehandelte Pflanzen } & 2,3 \pm 0,03, \\
3 \mu \text { g Gibberellin pro Pflanze } & 3,5 \pm 0,09, \\
9 \mu \text { g Gibberellin pro Pflanze } & 4,4 \pm 0,05 .
\end{array}
$$

Bei $17^{\circ}$ ergaben sich unter gleichen Lichtverhältnissen bei unbehandelten Pflanzen ein entsprechender Quotient von ebenfalls 2,3, bei den mit $9 \mu \mathrm{g}$ Gibberellin versetzten Pflanzen von 4,3. 
Im Dauerlicht kommt es während der Hauptwirkungszeit des Gibberellins bei Galinsoga parviflora sogar zu einer Aufrollung der Blätter. Das Gibberellin hat also hier eine stark schädigende Wirkung auf die Pflanzen. Dieser nachteilige Einfluß ist jedoch bei nur 8-stdg. täglicher Belichtung nicht zu beobachten. Gleichfalls nur im Dauerlicht wird eine Abnahme der Intensität der Grünfärbung der Blätter unter dem Einfluß des Gibberellins während seiner Hauptwirkungszeit deutlich, die wohl auf einem geringeren Chlorophyllgehalt beruhen dürfte. Eine hellere Färbung der Blätter wird auch als Symptom der durch den Pilz Gibberella fuiikuroi verursachten Krankheit genannt (S todola und Mitarbb. ${ }^{2}$ ). Die Aufrollung und die hellere Färbung der Blätter treten bei Galinsoga parviflora sowohl bei Gaben von $3 \mu \mathrm{g}$ als auch von $9 \mu \mathrm{g}$ Gibberellin pro Pflanze auf. Die Blätter, die sich sehr spät nach der Gibberellineinwirkung entwickeln, zeigen keine Aufrollung mehr.

Die Zahl der zu den einzelnen Zeitpunkten entwickelten Blattpaare ist bei Galinsoga parviflora durch Gibberellin etwas deutlicher gefördert als bei Agrostemma. Immerhin ist dieser Einfluß auch hier ziemlich schwach. Es ergaben sich 8 Tage nach Beginn der Gibberellin-Gaben folgende relative Werte (Tab. 5):

\begin{tabular}{|c|c|c|}
\hline $\begin{array}{c}\text { Gibberellinmenge pro } \\
\text { Pflanze (Galinsoga) } \\
{[\mu \mathrm{g}]}\end{array}$ & Dauerlicht & $\begin{array}{c}\text { 8 Stdn.Licht } \\
\text { täglich }\end{array}$ \\
\hline- & 100 & 100 \\
3 & 110 & 103 \\
9 & 116 \\
\hline
\end{tabular}

Tab. 5. (Erläuterung im Text).

\section{Wirkung auf die Blüten und Infloreszenzen}

Die Blüten und Knospen erscheinen unter der Gibberellin-Einwirkung früher als bei den unbehandelten Pflanzen. Die Gibberellin-Gaben verursachen bei Agrostemma githago im Dauerlicht folgende $\mathrm{Be}$ schleunigung des Erscheinens der Knospen:

$$
\begin{array}{ll}
\text { Bei } 26^{\circ}: & 1 \text { Tag, } \\
\text { bei } 17^{\circ}: & 3 \text { Tage, } \\
\text { bei } 10^{\circ}: & 4 \text { Tage. }
\end{array}
$$

Bei Galinsoga parviflora beträgt die entsprechende Beschleunigung:

$$
\begin{aligned}
& \text { Bei } 26^{\circ}: \quad 1 \text { Tag, } \\
& \text { bei } 17^{\circ}: \quad 3 \text { Tage. }
\end{aligned}
$$

Gaben von 3 und $9 \mu \mathrm{g}$ Gibberellin pro Pflanze bewirken bei beiden Arten die gleiche Beschleunigung des Erscheinens der Knospen gegenüber den Kontrollen.

Bei $26^{\circ}$ waren am 20. Tage nach Beginn der Behandlung bei Galinsoga bei den Kontrollen in Durchschnitt 0,5 Blütenstände bei einer Pflanze vorhanden. Bei Einwirkung von $3 \mu \mathrm{g}$ Gibberellin hatten sich bereits 1,7 und bei einer Gabe von $9 \mu \mathrm{g}$ Gibberellin 2,7 Blütenstände pro Pflanze entwickelt. Es ist also auch eine Differenzierung in der Zahl der Blütenstände zu bestimmten Zeitpunkten bei verschiedenen Gibberellingaben festzustellen.

Wie lange nach der Gibberellin-Behandlung bei Galinsoga unter Bedingungen, bei denen ein langsames Wachstum erfolgt, noch Unterschiede in der Zahl der Infloreszenzen einer Pflanze festzustellen sind, geht aus Tab. 6 hervor. Allerdings zeigt es sich auch hier, daß sich mit der Zeit die Unterschiede zwischen behandelten und unbehandelten Pflanzen ausgleichen.

Die Beschleunigung des Erscheinens der Knospen und Blüten ist im wesentlichen nur ein Ausdruck der raschen allgemeinen Entwicklung der Pflanzen unter dem Einfluß des Gibberellins. Die Zahl der Internodien und Knoten, über denen die ersten Blüten und Infloreszenzen erscheinen, ist bei beiden $\mathrm{Ar}$ ten bei den Kontrollen und den mit Gibberellin behandelten Pflanzen die gleiche.

Ein Einfluß des Gibberellins auf die Länge der Blütenblätter, Kelchblätter und die Anzahl der fertilen Samen in einer Frucht ist bei Agrostemma gi-

\begin{tabular}{|c|c|c|c|c|c|}
\hline & & \multicolumn{3}{|c|}{ Anzahl der Blütenstände } \\
pro Pflanze
\end{tabular}

Tab. 6 (Erläuterung im Text). 


\begin{tabular}{|c|c|c|c|}
\hline \multirow{2}{*}{$\begin{array}{c}\text { Gibberellin } \\
\text { pro Pflanze } \\
{[\mu \mathrm{g}]}\end{array}$} & \multicolumn{3}{|c|}{ Temperatur } \\
\cline { 2 - 4 } & $26^{\circ}$ & $17^{\circ}$ & $10^{\circ}$ \\
\hline- & 42,3 & 32,2 & 32,0 \\
3 & 41,5 & 23,1 & 25,2 \\
9 & 43,0 & 21,9 & 21,7 \\
\hline
\end{tabular}

Tab. 7. Zahl der Blüten in einem Blütenstand bei Calinsoga parviflora im Dauerlicht (bei $26^{\circ}$ Anzahl der sterilen Blüten; bei $17^{\circ}$ und $10^{\circ}$ Anzahl der fertilen Blüten; weitere Erläuterungen im Text).

thago nicht festzustellen. Bei Galinsoga parviflora ergibt sich dagegen im Dauerlicht eine deutliche Erniedrigung der Anzahl der fertilen Früchte in einem Blütenstand bei den mit Gibberellin behandelten Pflanzen (Tab.7). Die Gesamtzahl der Blüten in einem Körbchen bleibt dagegen die gleiche. Das ist aus den in Tab. 7 angeführten Zahlen für die Versuchsserien bei $26^{\circ} \mathrm{zu}$ ersehen. In dieser Temperatur werden nur sterile Blüten gebildet. Bei $17^{\circ}$ und $10^{\circ}$ wird dagegen der bei den Kontrollen hohe Anteil der fertilen Blüten durch Gibberellin-Gaben erniedrigt. Bei 8-stdg. täglicher Belichtung ist eine derartige Erniedrigung nicht festzustellen. Das entspricht der Erniedrigung der Assimilationsflächen und der Schädigung der Blätter, die nur im Dauerlicht bei den mit Gibberellin behandelten Pflanzen zu beobachten ist.

Herrn Prof. Dr. F. W. W e n t möchte ich auch an dieser Stelle besonders dafür danken, daß er mir die Durchführung der Arbeiten im Earhart Plant Research Laboratory ermöglichte.

\title{
Untersuchungen zur lichtabhängigen Phosphorylierung III
}

\author{
Die $\mathrm{CO}_{2}$-Abhängigkeit der ${ }^{32} \mathrm{P}$-Einlagerung in Ankistrodesmus \\ Von W. Simonis und H. Kating
}

Aus dem Botanischen Institut der Tierärztlichen Hochschule Hannover

(Z. Naturforschg. 11 b, 704-708 [1956]; eingegangen am 19. Juli 1956)

Die kurzfristige (5 Min.), unter Ausschluß von $\mathrm{CO}_{2}$ während der stationären Phase der Photosynthese, durchgeführte Einlagerung von mit ${ }^{2} 2 \mathrm{P}^{2}$ markierter Orthophosphorsäure in die einzellige Grünalge Ankistrodesmus wird unter den hier gewählten Versuchsbedingungen trotz der fast völligen Ausschaltung der $\mathrm{CO}_{2}$-Assimilation nicht verändert. Hoher $\mathrm{CO}_{2}$-Gehalt in der Gasphase setzt die festgestellte Phosphorylierung dagegen herab. Die Befunde werden unter Berücksichtigung der Photosynthese-Phosphorylierung (A r n o n) und einer besonderen, bei Belichtung wahrscheinlich auftretenden photo-sensibilisierten Phosphorylierung diskutiert.

$\mathrm{F}$ ür die Aufklärung des Zusammenhanges der lichtabhängigen Phosphorylierung der Pflanzen mit der Photosynthese ${ }^{1-3}$ erschien es erforderlich, die Abhängigkeit der Phosphorylierungs-Prozesse von der $\mathrm{CO}_{2}$-Assimilation und vom $\mathrm{CO}_{2}$-Gehalt der umgebenden Gasphase zu prüfen. Die bei Belichtung gefundene erhöhte Phosphorylierung ${ }^{1,4}$ könnte nämlich ihren Energiebedarf ganz oder teilweise aus dem Abbau von Intermediärprodukten der $\mathrm{CO}_{2}$-Assimilation entnehmen; vgl. die Zusammenstellung der theoretisch zunächst denkbaren Möglichkeiten des Ener-

1 W. S i m on is, Z. f. Naturforschg. 11 b, 354 [1956].

2 W. S i monis u. H. K a t ing, Z. Naturforschg. 11 b, 165 [1956].

3 W. Simonis u. E. Quensell, Naturwissenschaften 43, 204 [1956].

4 W. S im on is u. K. H. Grube, Z. Naturforschg. 7 b, 194 [1953].

5 O. K a n d l e r, Z. Naturforschg. 5 b, 423 [1950]; 10 b, 38 [1955].

6 O. W a rburg, Z. Elektrochem. angew. physik. Chem. 55, 447 [1951]; O. W a r burg u. G. Krip-

giegewinns für die lichtabhängige Phosphorylierung durch $\mathrm{Kandler^{5 }}$ sowie Warburg und Krippah $1^{6}$. Außerdem hängt die Entstehung der nach $\mathrm{Calvin}{ }^{7}$ bei der $\mathrm{CO}_{2}$-Reduktion gebildeten phosphorylierten Zwischenprodukte selbstverständlich vom $\mathrm{CO}_{2}$-Gehalt ab. Wintermans ${ }^{8}$ hat nun kürzlich aber in Versuchen, die sich über mehrere Stdn. erstreckten, bei Chlorella gefunden, daß die Umwandlung von Orthophosphat in zelleigene Phosphate im Licht in Gegenwart von einem $5 \% \mathrm{CO}_{2}$ enthaltenden Gasgemisch sogar langsamer abläuft als p a h 1, Z. angew. Chem. 66, 493 [1954].

7 M. C a l vi n, Conférences et Rapports, 3ème Congrès Internat. de Biochimie Bruxelles 1955, S. 211-224, Liège 1956.

8 J. F. G. M. W interm an s, Proc. Kon. nederl. Akad. v. Wetensch. Ser. C, 57, 574 [1954]; Meded. Landbouwhogeschool te Wageningen/Nederland 55, 69 [1955].

9 D. I. A rnon, M. B. All en, F. R. What ley, J. B. Capindale u. L. L. Ros en berg, Conférences et Rapports, 3ème Congrès Intern. de Biochimie Bruxelles 1955, S. 277, Liège 1956. 